\title{
Studies of the functional characteristics of adaptive X-ray optical elements based on combination of longitudinal and transverse acoustic waves in wide frequency ranges
}

\author{
A.E. Blagov ${ }^{1,2}$, A.V. Targonskiy ${ }^{1,2}$, V.I. Akkuratov ${ }^{1,2}$, Ya.A. Eliovich ${ }^{1,2}$, V.R. Kocharyan ${ }^{3,4}$, A.G. Kulikov ${ }^{1,2}$, \\ A.E. Movsisyan ${ }^{3,4}$, T.R. Muradyan ${ }^{3}$, Yu.V. Pisarevsky ${ }^{1,2}$, A.Yu. Seregin ${ }^{1,2}$, M.V. Kovalchuk ${ }^{1,2}$ \\ ${ }^{I} N R C$ "Kurchatov institute", 1, Akademika Kurchatova pl., Moscow, Russia, 123182, \\ ${ }^{2}$ FSRC "Crystallography and photonics" RAS, 59, Leninskii pr., Moscow, Russia, 119333, \\ ${ }^{3}$ Institute of Applied Problems of Physics NAS of Armenia, 25, Hrachya Nersissian Str., Yerevan, Republic of Armenia, 0014, \\ ${ }^{4}$ Tomsk Polytechnic University, 30, Lenin Avenue, Tomsk, Russia, 634050
}

Targonsky_AV@nrcki.ru

X-ray acoustic interactions allowing to implement the control of X-ray parameters are widely studied. Among the numerous researches, it is possible to highlight the ability of controlling the spatial and energy spectrum of X-ray radiation [1] and the effect of redistribution of intensity between transmitted and diffracted beam [2]. This paper describes the implementation of a combination of these two possibilities.

Fast control of X-ray parameters, including scanning diffraction conditions and controlling by times much shorter than possibilities of traditional approaches, is a very relevant scientific task. It will be shown that overcoming of limitation of traditional approach, such as complex goniometric systems, possible by using of non-mechanical adaptive X-ray optic elements, such as X-ray acoustic resonators of longitudinal oscillations or bimorph piezo-actuators. It allows fast and precise variation of X-ray diffraction parameters, varying the angular position of the X-ray beam and controlling its wavelength. Description of schemes and elements for fast tuning of beam parameters will be given.

The effects of the redistribution of intensities between the diffracted and transmitted X-ray beams under the conditions of excitation of resonant acoustic thickness oscillations in quartz crystals were investigated. It has been established that the effect of increasing the intensity of a diffracted beam almost linearly depends on the amplitude of ultrasound (the FWHM of the rocking curves does not change at the same time) and is observed for all the studied reflexes.

The time characteristics of the observed effects upon excitation and relaxation of ultrasonic oscillations were investigated for the first time: the process of increasing intensity takes about 250 microseconds, then its oscillation is observed for about 1 millisecond, and the process of complete relaxation takes about 1.5 milliseconds.

Design of elements combining thickness and longitudinal oscillations are considered, several schemes of implementation are proposed. For the first time, the distributions of the FWHM and peak intensities of the rocking curves in a quartz resonator in case of the simultaneous excitation of longitudinal and thickness oscillations were measured. It is shown that these two types of oscillations do not have a significant mutual influence. Therefore, this combination can be used to create universal adaptive elements of x-ray optics, which allow controlling the angular position and intensity of the diffracted beam simultaneously. The effect of intensity redistribution in Potassium and Rubidium hydrogen phthalate crystals, which are emerging materials for creating a two-frequency element, was studied for the first time.

Some results and prospects of implementation of such methods and elements at synchrotron radiation as well as laboratory sources will be discussed.

[1] A.E. Blagov, M.V. Kovalchuk et al. JETP letters, t.128, 5 (11) (2005). P.893

[2] A.P. Mkrtchyan, M.A. Navasardyan, V.K. Mirzoyan. JTP letters, 8, 677 (1982)

Keywords: X-ray diffraction, X-ray acoustooptics, Longitudinal oscillations, Transverse oscillations

The reported study was partially supported in the framework of the joint programs of the Russian Foundation for Basic Research (project № 18-52-05024 Arm_a and №18-32-20108 mol_a_ved) and Science Committee of Ministry of Education and Science of Armenia (project №18RF-142). 